\title{
Exophthalmos and corneal edema in a young ferret
}

\author{
Amy J. Funk, DVM, Tiffani D. Rogers, DVM, DACLAM, Rose Marie Dobbins \& Kelli Boyd, DVM, PhD, DAVCP
}

A technician discovered a 6-week-old male ferret with an eye abnormality during a morning health check. The ferret had been born in the research facility as part of a breeding colony maintained for influenza research and, prior to this observation, had had no ocular problems or developmental delays. Upon physical examination, a corneal opacity was noted in the left eye, as well as a diminished menace response. Other objective physical examination findings included a body temperature of $102.4^{\circ} \mathrm{F}$, body weight of $255 \mathrm{~g}$, a heart rate of 160 beats/min, and a respiratory rate of 36 breaths $/ \mathrm{min}$. The animal was adequately hydrated, its mucous membranes were pink, and its capillary refill time was less than 3 s. Activity and appetite were unaffected and all other physical parameters were within normal limits.

The ferret shared a modified rabbit cage (Allentown, Allentown, NJ) with two littermates, both of which appeared healthy with no ophthalmic abnormalities. Neither parent of the 6-week-old male ferret had a history of ocular disease. The initial clinical assessment was idiopathic corneal edema, possibly from an immature cataract. No treatment was initiated at that time and the animal was monitored closely for any changes.

Two weeks later, the attending husbandry technician reported acute changes to the left eye. Upon reexamination, the left eye was markedly larger and the ferret lacked a menace response. The acute progression of clinical signs necessitated that the animal be euthanized and submitted for necropsy.

On gross examination, unilateral exophthalmos and corneal opacity in the left eye were the only abnormalities found in the ferret (Fig. 1). Both eyes were collected and fixed in $10 \%$ neutral buffered formalin, processed routinely, embedded in paraffin, and sectioned at $4 \mu \mathrm{m}$. Histologic findings included thin sclera, corneal edema, anterior synechia (Fig. 2), and inner retinal atrophy (Fig. 3). The anterior synechia were broad and precluded a detailed examination of the filtration angle. Retinal atrophy consisted of the loss of the ganglion cells, the inner plexiform layer, and a thin inner nuclear layer.

Figure 1 shows an obvious size difference between the right and left eye. What condition is consistent with these histological results? Is this ocular condition

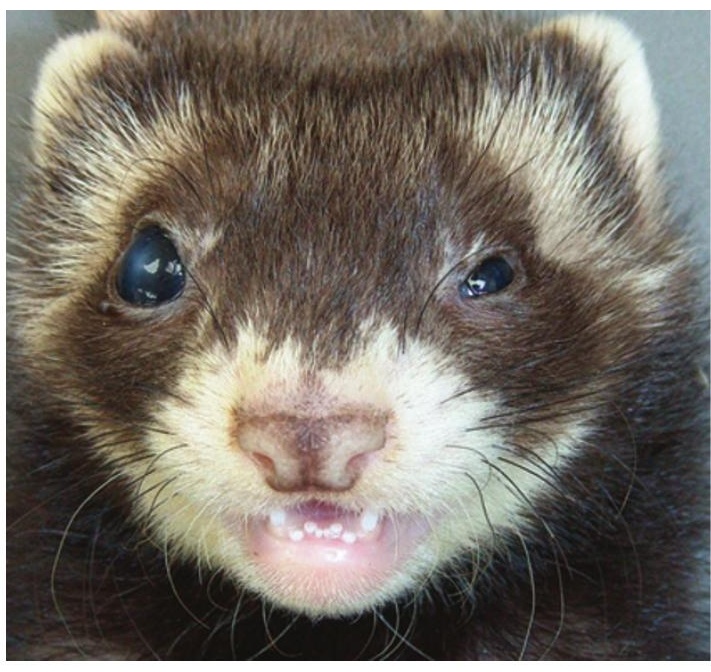

FIGURE 1 | Unilateral exophthalmos and corneal edema in the left eye of an 8-week-old male ferret.

commonly seen in ferrets? What are other possible ocular maladies? Are any of these conditions related?

\section{What's your diagnosis?}

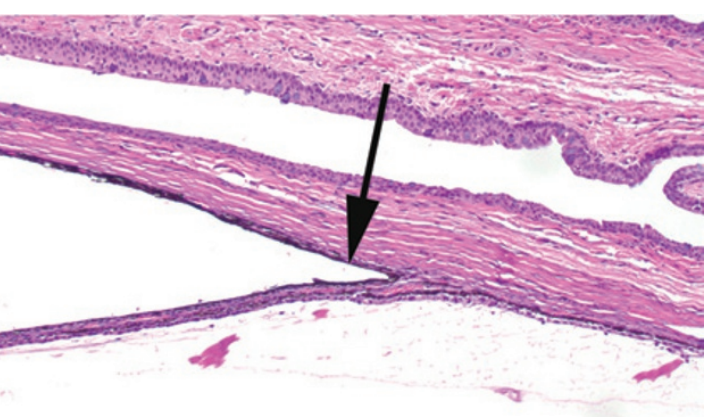

FIGURE 2 | Photomicrograph of the left eye of the ferret in Figure 1 showing the anterior chamber of the eye. (H\&E stain, $\times 100$ magnification.)

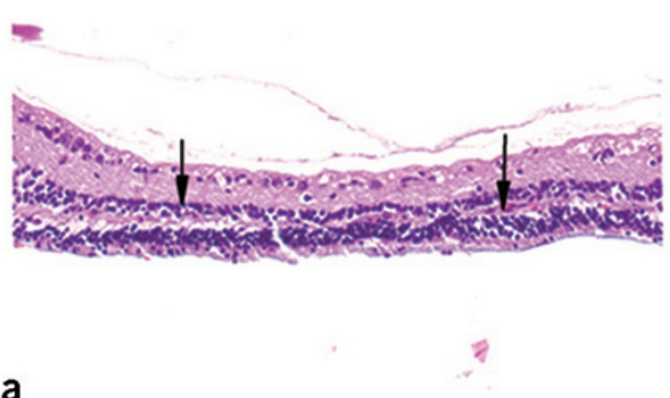

FIGURE 3 | Photomicrograph of the left eye of the ferret in Figure 1 showing the retina. There is marked depletion of the inner layers in the affected retina (a) compared with the retina of the unaffected right eye (b). (H\&E stain, $\times 200$ magnification.) 